\title{
A Set of Customizable Games Supporting Therapy of Children with Cerebral Palsy
}

\author{
Beant Dhillon, Areti Goulati, Ioannis Politis, \\ Agata Raczewska, and Panos Markopoulos \\ Eindhoven University of Technology, Den Dolech 2, 5612 AZ \\ Eindhoven, the Netherlands \\ \{B.K.Dhillon, A.Goulati, I.Politis, \\ A.M.Raczewska, P.Markopoulos\}@tue.nl
}

\begin{abstract}
This research explores the potential of tangible tabletop technology for motor skills training of children with Cerebral Palsy. Therapists have emphasized the importance of customization of therapy programs to the needs of each patient. Five customizable games for the TagTiles tangible interactive gaming board are presented. These games have been developed based on feedback from 11 therapists from two clinics in the Netherlands, 9 children with Cerebral Palsy as well as 14 healthy children. The design process and the potential of this solution are briefly outlined.
\end{abstract}

Keywords: Cerebral Palsy, Motor Skills, Rehabilitation, TagTiles, Therapeutic Games, Design.

\section{Introduction}

Cerebral Palsy (CP) is a term describing a set of neurological disorders that cause physical disability, mainly affecting body movements and muscle coordination. In additional to traditional means of therapy, tabletop interactive surfaces offer a lot of potential. The TagTiles board [1] is a programmable grid of RFID sensors, able to provide visual and sound feedback, which is triggered by objects with attached RFID tags. The use of TagTiles in training for children with $\mathrm{CP}$ has been explored in the past [2] resulting in a series of games that support the training of arm-hand skills.

However, that work did not address several requirements that relate to the use of the games in actual therapy. Interviews with therapists emphasized the importance of supporting customization to each child's needs, and flexibility: therapists need to interrupt games and switch to other ones fluently during a therapy session.

\section{CP Game Design}

Input collected over 4 sessions with 11 therapists and 9 children with upper extremities CP from 2 different Dutch clinics resulted in the design of five new games and accompanying objects. These train different hand movements; grasping, wrist supination, and finger, shoulder and wrist extension. Objects of varying shapes, sizes, 


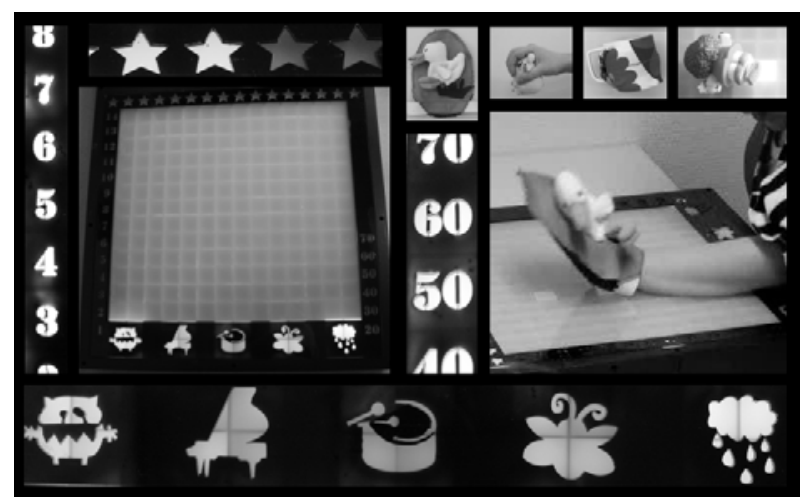

Fig. 1. The TagTiles board (left), the stars earned (top left), the game menu (bottom), the game speed indication (extreme left), the repetitions indication (middle), some of the objects designed (top right) and a child with $\mathrm{CP}$ interacting with the board (right)

textures and weights have been used along with adjustable Velcro tags (see Fig.1). All objects have been designed so as to prevent compensation.

The interaction in all games involves matching the flashing squares on the board with the objects in the child's hand. Every game uses an engaging background story (e.g., feeding a hungry monster or playing the piano). The therapists can control the difficulty level (i.e. game speed and number of repetitions) through a specially designed menu. To maintain motivation, encouraging feedback is provided through sounds and music. At the end of the game, feedback regarding performance is provided in terms of 'stars' earned by the child.

An evaluation session with 14 healthy children was performed as well in order to assess the very important fun aspect of the games. The games are now fully functional and ready to be embedded in a therapy setup.

\section{References}

1. Verhaegh, J., Fontijn, W., Hoonhout, J.: TagTiles: optimal challenge in educational electronics. In: 1st International Conference on Tangible and Embedded Interaction, pp. 187-190. ACM, New York (2007)

2. Li, Y., Fontijn, W., Markopoulos, P.: A tangible tabletop game supporting therapy of children with Cerebral Palsy. In: Fun and Games 2008: Second International Conference, pp. 182-193. Springer, Berlin (2008) 